\title{
BEAM LOSS SCENARIOS AND STRATEGIES FOR MACHINE PROTECTION AT THE LHC
}

R. Schmidt, R. Assmann, H. Burkhardt, E. Carlier, B. Dehning, B. Goddard, J.B. Jeanneret, V. Kain, B. Puccio, J. Wenninger

\begin{abstract}
At the Large Hadron Collider (LHC) with nominal parameters at $7 \mathrm{TeV}$, each proton beam has an energy of more than $330 \mathrm{MJ}$ threatening to damage accelerator equipment in case of uncontrolled beam loss. To prevent such damage, kickers are fired in case of failure deflecting the beams into dump blocks. The dump blocks are the only elements that can safely absorb the beams without damage. The time constant for particle losses depends on the specific failure and ranges from microseconds to several seconds. Starting with some typical failure scenarios, the strategy for the protection during LHC beam operation is illustrated. The systems designed to ensure safe operation, such as beam dump, beam instruments, collimators / absorbers and interlocks are discussed.
\end{abstract}

AB Division

Presented at the HALO'03 ICFA Advanced Beam Dynamic Workshop, Montauk, NY, USA

May $19-23,2003$

CERN

CH - 1211 Geneva 23

Switzerland

Geneva, 22'August 2003 


\title{
Beam loss scenarios and strategies for machine protection at the LHC
}

\author{
R.Schmidt, R.Assmann, H.Burkhardt, E.Carlier, B.Dehning, B.Goddard, \\ J.B.Jeanneret, V.Kain, B.Puccio, J.Wenninger
}

CERN, Switzerland, Geneva

\begin{abstract}
At the Large Hadron Collider (LHC) with nominal parameters at $7 \mathrm{TeV}$, each proton beam has an energy of more than $330 \mathrm{MJ}$ threatening to damage accelerator equipment in case of uncontrolled beam loss. To prevent such damage, kickers are fired in case of failure deflecting the beams into dump blocks. The dump blocks are the only elements that can safely absorb the beams without damage. The time constant for particle losses depends on the specific failure and ranges from microseconds to several seconds. Starting with some typical failure scenarios, the strategy for the protection during LHC beam operation is illustrated. The systems designed to ensure safe operation, such as beam dump, beam instruments, collimators / absorbers and interlocks are discussed.
\end{abstract}

\section{INTRODUCTION}

To deliver proton-proton collisions at the centre of mass energy of $14 \mathrm{TeV}$ with a nominal luminosity of $10^{34} \mathrm{~cm}^{-2} \mathrm{~s}^{-1}$, the LHC will operate with high-field dipole magnets using $\mathrm{NbTi}$ superconductors cooled below the $\lambda$-point of helium. The most important parameters for the LHC as proton collider are given in Table 1. One of the main challenges is the safe operation with beam parameters pushed to the extreme in order to achieve design luminosity. Whereas the proton energy is a factor of seven above accelerators such as SPS, Tevatron and HERA, the energy stored in the beams is more than a factor of 100 higher. For damage of equipment the transverse energy density is the relevant parameter, a factor of 1000 higher.

The beams must be handled in an environment with superconducting magnets that could quench in case of fast losses of $10^{-8}-10^{-7}$ of the nominal number of protons (at $7 \mathrm{TeV}$ ). This value is orders of magnitude lower than for any other accelerator with superconducting magnets. Any uncontrolled release of the beam energy could cause serious damage to equipment. As an example, a fast loss of about $5 \%$ of a single nominal $7 \mathrm{TeV}$ bunch in one spot could already damage material with high $\mathrm{Z}$, such as copper.

\begin{tabular}{|l|c|c|}
\hline \multicolumn{3}{|c|}{ TABLE 1: LHC Parameters } \\
\hline Energy at collision & 7 & $\mathrm{TeV}$ \\
\hline Dipole field for 7 TeV & 8.33 & $\mathrm{~T}$ \\
\hline Luminosity & $10^{34}$ & $\mathrm{~cm}^{-2} \mathrm{~s}^{-1}$ \\
\hline Protons per bunch & $1.1 \cdot 10^{11}$ & \\
\hline Number of bunches / beam & 2808 & \\
\hline Nominal bunch spacing & 25 & $\mathrm{~ns}$ \\
\hline Normalised emittance & 3.75 & $\mu \mathrm{m}$ \\
\hline Beam size at IP for 7 TeV & 15.9 & $\mu \mathrm{m}$ \\
\hline $\begin{array}{l}\text { Typical beam size in arcs (rms) } \\
\text { for 7 TeV }\end{array}$ & 300 & $\mu \mathrm{m}$ \\
\hline Arc magnet coil inner diameter & 56 & $\mathrm{~mm}$ \\
\hline
\end{tabular}

Other accelerators operate with beams that have much less stored beam energy but where protection of equipment from beam losses is still a concern. For example, the SPS beam drilled holes through the vacuum chamber when the protection systems did not work correctly. Both at SPS and TEVATRON radiation damage of silicon detectors of the experiments occurred after beam losses.

\section{CERN}

CH - 1211 Geneva 23

Switzerland

Geneva, 22'August 2003 


\section{EUROPEAN ORGANIZATION FOR NUCLEAR RESEARCH \\ European Laboratory for Particle Physics}

\section{BEAM LIFETIME}

Under optimum condition without collision the beam lifetime could exceed, say, $100 \mathrm{~h}$. This would be very comfortable since the beam deposited power into the equipment is only about $1 \mathrm{~kW}$. If the lifetime decreases to $10 \mathrm{~h}$, the LHC would rely on collimators to capture the losses in two cleaning insertions with normal conducting magnets [1]. The collimation system is designed to accept a lifetime of about $0.2 \mathrm{~h}$ for a $10 \mathrm{~s}$ long transient, e.g. when changing the betatron tune. This corresponds to a power deposition of $500 \mathrm{~kW}$. If the lifetime becomes even smaller, in particular after equipment failure, the beams will have to be dumped immediately. Depending on the type of failure, dumping the beams must be very fast (see Table 2). The dump blocks are the only elements that can absorb the energy stored in the LHC beam without being damaged.

\begin{tabular}{|l|l|l|}
\hline \multicolumn{3}{|c|}{ Table 2: Lifetime of the LHC beams } \\
\hline $\begin{array}{l}\text { Beam } \\
\text { lifetime }\end{array}$ & $\begin{array}{l}\text { Beam power into } \\
\text { environment } \\
(1 \mathrm{beam})\end{array}$ & Comments \\
\hline $100 \mathrm{~h}$ & $1 \mathrm{~kW}$ & Healthy operation \\
\hline $10 \mathrm{~h}$ & $10 \mathrm{~kW}$ & $\begin{array}{l}\text { Operation acceptable, } \\
\text { collimation must absorb } \\
\text { large fraction of beam energy }\end{array}$ \\
\hline $12 \mathrm{~min}$ & $500 \mathrm{~kW}$ & $\begin{array}{l}\text { Operation only possibly for } \\
\text { short time, collimators must } \\
\text { be very efficient }\end{array}$ \\
\hline $1 \mathrm{~s}$ & $330 \mathrm{MW}$ & $\begin{array}{l}\text { Failure of equipment - beam } \\
\text { must be dumped rapidly }\end{array}$ \\
\hline 15 turns & Several $100 \mathrm{GW}$ & $\begin{array}{l}\text { Failure of D1 normal } \\
\text { conducting dipole magnet - } \\
\text { detect beam losses, beam } \\
\text { dump as fast as possible }\end{array}$ \\
\hline 1 turn & $\sim$ TW & $\begin{array}{l}\text { Failure at injection or during } \\
\text { beam dump, potential } \\
\text { damage of equipment, } \\
\text { passive protection relies on } \\
\text { collimators }\end{array}$ \\
\hline
\end{tabular}

\section{COLLIMATORS AND BEAM ABSORBERS}

Three of the eight insertions are dedicated to machine protection. One insertion is for the beam dump systems, one with collimators capturing protons with large betatron amplitudes, and a third with collimators in locations with non-zero dispersion catching protons with large momentum deviations. Additional collimators are installed in most other insertions.

In order to limit the beam losses in superconducting magnets, the LHC will be the first machine requiring collimators to define the mechanical aperture through the entire cycle. For efficient beam cleaning, the collimators are adjusted between 5-9 $\sigma$. For operating at $7 \mathrm{TeV}$, the opening between two collimators jaws is about 3-4 $\mathrm{mm}$. More than $99.9 \%$ of the protons in the beam halo should be captured in the cleaning insertions. The elements that are closest to the beam are the collimator jaws. In case of failures causing fast movement of the beam, the jaws intercept particles and prevent damage of other equipment until the beams are safely extracted.

\section{FAILURE SCENARIOS AND PROTECTION SYSTEMS}

Most likely are failures in the magnet and powering system, with about 8000 magnets powered in 1700 electrical circuits. Other failures are due to aperture restrictions, with two beams circulating through $53 \mathrm{~km}$ of beam vacuum pipe with the beam screen inside, helium feedthroughs, interconnects, RF shielding etc. Many vacuum valves and more than 100 collimator jaws could also obstruct the beam passage.

Beam losses due to a failure can be in a single turn, or during many turns:

- One turn failures: ultra-fast losses

- Multiturn failures: very fast losses in less that $5 \mathrm{~ms}$, and fast losses in more than $5 \mathrm{~ms}$

- Steady losses (one second or more), discussed in the next chapter

\section{One-turn failures (ultra-fast losses)}

Beam can be lost in less than one turn due to a failure at injection or due to a failure when extracting the beam into the dump blocks.

Injection: The beam is accelerated in the SPS to $450 \mathrm{GeV}$ and then sent through $\sim 2.8 \mathrm{~km}$ long transfer lines to the LHC [2]. During extraction from the SPS, beam transfer and injection into the LHC, the beam could damage septum magnets or kickers.

There could also be a failure by septum magnet or injection kicker that steers the beam onto a wrong trajectory. In order to make the beam travel correctly through the first turn, the parameters of all magnets in the LHC should be set to the correct values. If one of the magnets had a wrong current value, or in case of an aperture restriction (for example due to a closed vacuum valve), the beam would be lost. Assuming the worst case that a corrector magnet close to one of the

CERN

CH - 1211 Geneva 23

Switzerland

Geneva, 22 August 2003 


\section{EUROPEAN ORGANIZATION FOR NUCLEAR RESEARCH}

European Laboratory for Particle Physics

experiments is set to maximum current, the beam would go straight into the detector.

In order to avoid damage during injection it is proposed to ensure correct beam parameters in the SPS before extraction ("beam quality check"). If the parameters were not acceptable, the beam would not be extracted. Collimators in the transfer line will capture bunches that are outside the acceptable trajectory range, to avoid damage of elements in the injection region and the following sectors of the LHC [3]. Additional collimators downstream of the kicker magnet will prevent damage in the LHC in case of a mis-kicked beam.

With no beam circulating in the LHC, only injection of beam with limited intensity (=non destructive) is allowed. Beam exceeding this intensity can only be injected when there is already beam circulating [4]. The damage level at $450 \mathrm{GeV}$ is about 10-20 bunches with nominal intensity.

Extraction: For a clean extraction of the beam into the dump blocks several conditions have to be met [5]:

- The beam dump kicker must by synchronised with the $3 \mu$ s long particle free abort gap.

- Since the aperture of the dump channel is tight, closed orbit errors in the dump insertion must be limited to about $4 \mathrm{~mm}$.

- Energy tracking between the main dipole magnets and the elements in the beam dump system (kickers and septa magnets) must be ensured by special hardware with ultrahigh reliability.

A likely failure scenario is the pre-firing of one beam dump kicker module. The other 14 kicker modules would be immediately triggered after such failure, but about 94 bunches deflected up to $4 \mu$ s after the pre-firing would not be extracted correctly. The bunches having received the smallest kick would travel through the machine, come back after one turn and then be deflected by the beam dump kicker in the second turn. These bunches could have a large offset at the second deflection, and absorbers must ensure that no equipment is damaged. About 10 bunches would receive a kick such that they reach the cleaning insertion and hit the collimators. Bunches that are deflected with a larger angle would hit an absorber in the dump insertion (TCDQ). Some 40 bunches would hit an absorber in front of the septum magnet (TCDS).

A failure in the synchronisation between RF and abort gap has slightly less severe consequences, since the number of bunches that do not travel correctly through the beam dump channel is smaller.
The design of collimators and absorbers is a major challenge. The objects should not be damaged in case of an impact of about 10-20 bunches [1]. To stand such an event, the material for the collimators, originally $\mathrm{Al}$ and $\mathrm{Cu}$, is being reconsidered.

During extraction, the $3 \mu$ s long gap must be free of particles. Debunching caused by RF noise, intrabeam scattering, etc. populates the beam abort gap [1]. At an energy of $7 \mathrm{TeV}$, non-captured protons will lose energy by synchrotron radiation and therefore be captured in the momentum cleaning insertion.

Whenever a failure during extraction occurs, bunches will oscillate with large amplitudes around the closed orbit. Orbit excursions exceeding, say, $4 \mathrm{~mm}$ limiting the aperture could lead to damage in case of unclean beam dump. Hence, the closed orbit around the machine must be well controlled [6].

\section{Multiturn failures (fast / very fast losses)}

Failures that could drive the beam unstable are mainly quenches of superconducting magnets, of a single magnet or several magnets. The current decay in the quenching magnet is approximately Gaussian, from the maximum (corresponding to the initial current) down to zero current. Other hardware failures require fast discharge of magnets with an exponential current decay. There could also be a failure in the power converter control - for example the power converter ramps current with maximum voltage, or a wrong reference value for the current. An electric short in the coil of a normal conducting magnet is also considered. After a failure in the RF system, the beam is dumped immediately, since the beam would debunch and the abort gap would be filled with particles. A clean dump would no longer be possible. There are operational failures, and combined failures (for example after Mains disturbances).

A failure of a normal conducting magnet in two insertions with physics experiments (ATLAS and CMS) is most critical. The deflection by the D1 magnet installed at a location with a $\beta$-function of more than $4000 \mathrm{~m}$ leads to a fast change of the closed orbit. In [7], several failures were considered:

- Powering failure of the normal conducting dipole magnets D1 for beam separation at $\beta=4000 \mathrm{~m}$

- Quench of the superconducting dipole magnets for beam separation at $\beta=2000 \mathrm{~m}$

- Quench of the very strong low- $\beta$ triplet quadrupole magnets at $\beta=4000 \mathrm{~m}$ with orbit

CERN

CH - 1211 Geneva 23

Switzerland

Geneva, 22'August 2003 


\section{EUROPEAN ORGANIZATION FOR NUCLEAR RESEARCH \\ European Laboratory for Particle Physics}

offset due to the crossing angle. This will also lead to a change of tunes and betatron functions - Quenches of superconducting arc dipole magnets

Since the collimators are very close to the beam, protons in the tails of the distribution would touch the jaws already after several turns. A failure of the normal conducting D1 magnets leads to losses within the shortest time. The loss would exceed more than $10^{9}$ protons after about 15 turns, and possibly damage the collimators after 30 turns (assuming that the collimators can withstand a beam loss of about $10^{12}$ protons). In Fig.1, the change of closed orbit after a failure of $\mathrm{D} 1$ at the position of three different collimators is given (lower part of the figure). In the quadrupoles, etc.) would be detected. The beam is dumped if the threshold is exceeded. It is also proposed to detect rapid beam position changes. If beam orbit movements exceed a predefined value, say, about $0.2 \mathrm{~mm} / \mathrm{ms}$, the beams are dumped. Such a system could detect failures earlier than beam loss monitors and is a redundant system for protection.

Fast Losses: Beam loss monitors around the machine and signals from equipment in case of hardware failure for many systems will be used to generate beam dump requests and complement fast beam loss and beam position monitors.

Steady losses: the beam losses and heat load at collimators will be monitored. A beam dump in case of unacceptable lifetime is also being considered.

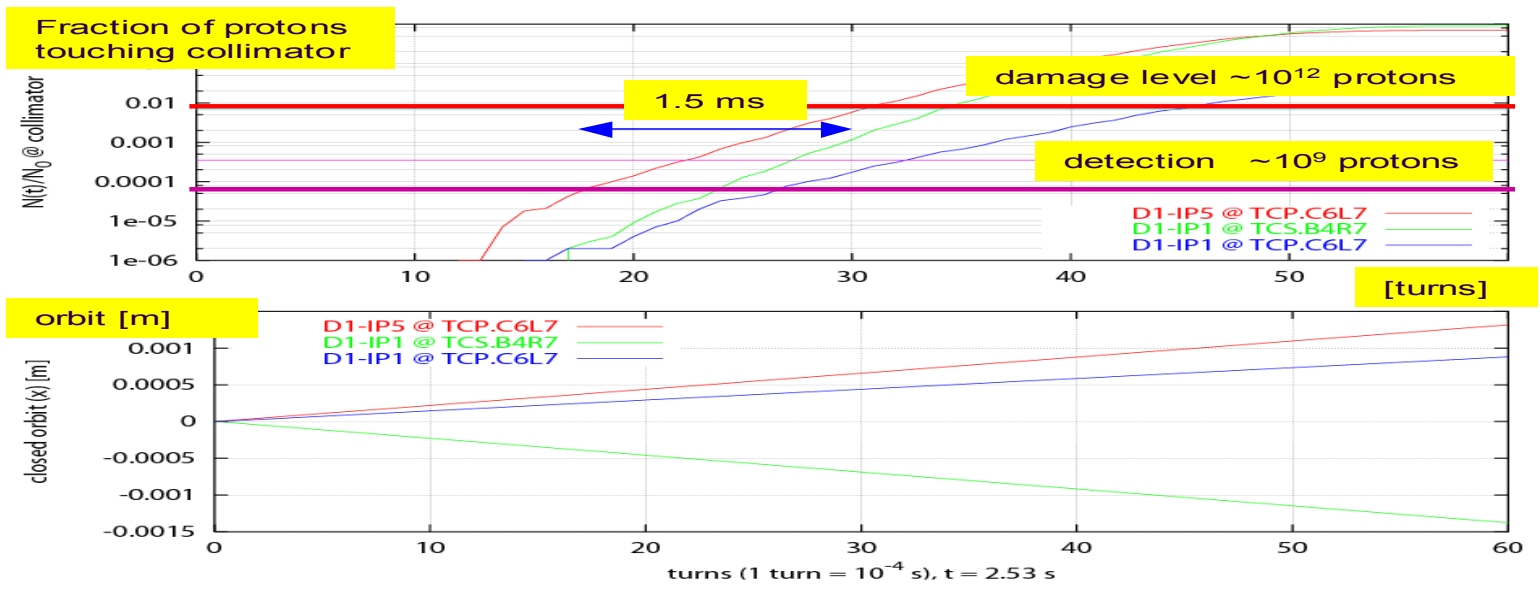

Fig.1: Beam losses (upper curve) and orbit movement (lower curve) after a failure of the D1 magnets as function of the number of turns. Three collimators that are affected most by a failure have been considered.

upper part the number of protons touching the collimators is shown for a Gaussian particle distribution. If the particle distribution is not Gaussian, the time between detection of the loss and damage of the collimators could be even shorter.

\section{STRATEGIES FOR PROTECTION}

Ultrafast losses: The hardware of injection and extraction system should be as reliable as possible. However, it is known from other accelerators that such failures cannot be completely excluded. Protection relies on collimators and beam absorber that need to be correctly positioned with respect to the beam to capture the particles.

Very fast losses: For failures that lead to very fast beam movements, the beam losses close to aperture limitations (collimators, beam absorbers, low-beta
Other options: During the most critical part of operation at $7 \mathrm{TeV}$ with squeezed optics to $\beta^{*}=0.5 \mathrm{~m}$, the triplet magnets focusing the beams into the interaction point have a very tight aperture. Absorbers in front of the triplet magnets would protect the superconducting quadrupoles in case of failure.

Critical elements for the protection of the LHC are the TCDQ collimators in the dump insertion. The TCDQ must be adjusted to about $10 \sigma$ from the beam. For protection against dump failures a jaw on one side would be sufficient. However, it is suggested to install absorbers from two sides in order to avoid a large orbit offset that would compromise the TCDQ functionality.

As it has been shown, a failure of the D1 magnets would lead to very fast orbit changes. Increasing the inductance in the electrical circuit with D1 magnets by about a factor of five, possibly with a superconducting solenoid in series with the magnets, would increase the

CERN

CH - 1211 Geneva 23

Switzerland

Geneva, 22'August 2003 


\section{EUROPEAN ORGANIZATION FOR NUCLEAR RESEARCH \\ European Laboratory for Particle Physics}

time constant for orbit changes and relax the parameters for the protection system.

\section{REFERENCES}

1. Jeanneret, J.B. et al., "Collimation at LHC", these proceeding and Assmann, R. et al, "Designing and Building a Collimation System for the High-Intensity LHC Beam”, PAC 2003, Portland, USA, May 2003

2. Hilaire, A. et al., "Beam Transfer to and injection onto the LHC”, EPAC '98, Stockholm, June 1998

3. Burkhardt, H., "Do we need collimation in the transfer lines?" in Chamonix XI, March 3-8, 2003, CERN-AB2003-008 ADM, April 2003

4. Schmidt, R. and Wenninger, J., "LHC Injection Scenarios”, LHC Project Note 287, CERN, March-2002

5. Goddard, B., "Apertures During Beam Abort", in Chamonix XI, March 3-8, 2003, CERN-AB-2003-008 ADM, April 2003

6. Wenninger, J., "Orbit Control for Machine Operation and Protection", in Chamonix XI, March 3-8, 2003, CERNAB-2003-008 ADM, April 2003

7. Kain, V., "Studies of equipment failures and beam losses in the LHC", Diploma thesis, Wien, October 2002

CERN

CH - 1211 Geneva 23

Switzerland

Geneva, 22'August 2003 